\title{
Evaluation of a Rapid Diagnostic Test Currently Being Used in the Management of Malaria in Patients of the Hia/ Chu-Parakou and the Boko Zone Hospital in Northern Benin
}

\author{
Tokponnon $\mathrm{F}^{1 *}$, Osse $\mathbf{R}^{1}$, Houessou $\mathrm{C}^{2}$, Akogbeto \\ $M^{1}$ and Kinde-Gazard $D^{3}$ \\ ${ }^{1}$ Cotonou Entomological Research Center, Benin \\ ${ }^{2}$ Superior School Falcon, Benin \\ 3Parasitology-Mycology Research Unit of the Faculty of \\ Health Sciences of Cotonou, Benin
}

*Corresponding author: Filémon Tokponnon, Cotonou Entomological Research Center, Benin

Received: July 27, 2021; Accepted: August 14, 2021; Published: August 21, 2021

\begin{abstract}
Parasitological diagnosis is a fundamental element in the adequate management of the disease. In the last decade, there has been a resurgence of interest in the development of malaria Rapid Diagnostic Test (RDT) kits. This is the case with SD Bioline Malaria Ag Pf, which searches for HRP2 antigen by immunochromatography. The objective of this study is to compare the results of RDT SD Bioline Malaria Ag Pf in use with the results of Thick Gout (TG) in the biological diagnosis of malaria.

This was a cross-sectional, descriptive and evaluative study carried out at the Hôpital d'Instruction des Armées-Center Hospitalier et Universitaire de Parakou and at the Boko zone hospital from April 20 to July 30, 2015. Patient identification, we used the non-probabilistic method and the convenience choice technique. The study involved 503 patients.

The results of this study showed a good performance of the RDT SD Bioline Malaria Ag Pf. Among the 503 patients, 199 or $39.6 \%$ were positive for the RDT against 180 or $35.8 \%$ positive for the Thick Gout (TG). Sensitivity, specificity, positive and negative predictive values of the test compared to the thick film were respectively $(91.7 \%, 89.5 \%, 82.9,95)$ and the Kappa coefficient of 0.88 testifies a good match. False positive cases are noted in patients on treatment and even after recovery due to the persistence of the HRP2 antigen in the blood. However, it constitutes an interesting alternative to the management of malaria. At the end of this study, we suggest continuing the use of RDTs in health centers where microscopy is absent and/or reinforcing microscopy, and to strengthen staff training in the management of malaria cases.
\end{abstract}

Keywords: Evaluation; Rapid diagnostic test; HRP2; Thick gout; Benin

\section{Introduction}

Malaria, a potentially fatal parasitic disease, remains a serious public health problem, particularly in African countries [1].

Globally, approximately 300 to 500 million people suffer from malaria in a year, causing 1.5 to 2.7 million deaths per year. According to the World Health Organization (WHO 2012), "malaria continues to weigh unacceptably on the health and economic development of more than 100 countries around the world and every 30 seconds a child dies from malaria" $[2,3]$. Malaria control strategies are directed against both the parasite and the vector. Its management requires a good diagnosis and treatment with an antimalarial drug adapted to the level of progression of the disease. The diagnosis of malaria is based on clinical criteria (clinical diagnosis), confirmed by the demonstration of haematozoa or their antigens/antibodies in the blood (laboratory diagnosis). The diagnosis and treatment of malaria have long been based on clinical evidence without laboratory confirmation [1,4]. But Since 2010, the WHO recommends parasitological confirmation of the diagnosis of malaria before treatment for all age groups [5]. The use of rapid diagnostic tests for antigens is an essential part of this strategy and the mainstay of expanding access to malaria diagnosis, as they allow parasitological diagnosis in areas where it is impossible to maintain. Good microscopy services [6].

In order to ensure the compulsory diagnosis of all suspected cases of malaria at the level of health facilities, Benin has adopted in its malaria control policy, the systematic confirmation of cases before any treatment using an antimalarial drug. In 2011, the national malaria management guidelines adopted by the NCMP and all the authors recommend both microscopy and Rapid Diagnostic Tests (RDTs) to be used at all levels of the health pyramid. As diagnostic tools to effectively implement this policy with the aim of early and correct management of malaria cases and prevention of resistance to antimalarials [7]. Indeed, an early diagnosis allows the rapid implementation of the treatment; shortening the duration of the infestation while preventing complications, including most deaths from severe forms. These tests are most used in peripheral health centers that do not have a laboratory for good case management or to meet medical emergencies $[1,2,4]$. Several studies [8-10] have shown the relevance of these RDTs in the diagnosis of malaria, but it is
Austin J Infect Dis - Volume 8 Issue 4 - 2021

Submit your Manuscript | www.austinpublishinggroup.com

Tokponnon et al. (C) All rights are reserved
Citation: Tokponnon F, Osse R, Houessou C, Akogbeto M and Kinde-Gazard D. Evaluation of a Rapid Diagnostic Test Currently Being Used in the Management of Malaria in Patients of the Hia/Chu-Parakou and the Boko Zone Hospital in Northern Benin. Austin J Infect Dis. 2021; 8(4): 1057. 
important to assess the performance of these diagnostic tools during their use. Tests performed with Plasmodium falciparum Protein Rich in Histidine 2 (PfHRP2) antigens are currently over $90 \%$ of malaria RDTs used worldwide [11]. The Boko zone hospital in Parakou is a center where malaria treatment is free for children aged 0 to 5 and for pregnant women according to the strategy decreed by the government of Benin. RDTs are used daily in addition to GE in the diagnosis of malaria [12-16]. It has a high attendance by the disadvantaged social classes, residing nearby but also in districts of the city of Parakou. The Army Instruction Hospital (HIA) of Parakou, Hospital and University Center is a new benchmark health facility in the north of the country. Would the use of RDTs in this new referral center not allow good management of malaria by reinforcing the existing microscopy? However, what then is the performance of RDTs in use in Parakou health centers? To address these concerns, we proposed to carry out this study to assess the performance of RDTs in these two health structures in Parakou [17-20]. The general objectives of this study are to evaluate the performance of the RDT SD BIOLINE Malaria Ag Pf in the treatment of malaria at the HIA of Parakou and at the Boko zone hospital. Specifically, it will be a question of (i) describing the results of RDT SD Bioline Malaria Ag Pf and thick gout in the laboratory diagnosis of malaria at the HIA and at the Boko zone hospital [21-25].

\section{Materials and Methods}

\section{Study framework}

Our study was carried out in two Laboratories: the Army Instruction Hospital of Parakou and the Boko Zone Hospital mainly in the Parasitology section over a period of four months from April to July 2015.

Army instruction Hospital-Hospital and University center of Parakou (HIA/CHU/PKOU): Located at the entrance to the city of Parakou, about three kilometers from the city center, more precisely in the Sinagourou 3 district; The Army Instruction Hospital-Hospital and University Center (HIA-CHU/PKOU) is built on a total area of seven thousand four hundred six (7406) square meters by the Chinese hence its common name "Chinese hospital". It was inaugurated on February 13, 2011. The result of Sino-Beninese cooperation, the HIA$\mathrm{CHU} / \mathrm{PKOU}$ is a reference hospital with military status open to the public.

The different services of the HIA: The Army Instruction HospitalHospital and University Center (HIA-CHU/PKOU) is currently an integral part of the university hospital space of Benin with the advent of the Faculty of Medicine of the University of Parakou and must now face three essential missions, namely: the care mission, the training mission and the research mission.

This center has several services spread over three main sectors:

- The medical sectors include the following services: emergencies, resuscitation, internal medicine, surgery, ophthalmology, obstetrician gynecology, stomatology, odontostomatology, cardiology, pharmacy, operating room, and social service.

- The administrative, economic and financial sectors.

- The medico-technical sectors concern: radiology, the ultrasound unit, pathological anatomy.
- The biomedical analysis laboratory, which represents my study framework itself made up of several sections including biochemistry; parasitology; serology; hematology and bacteriology.

Boko Zone Hospital: It is located in the Parakou - N'Dali sanitary zone and about $15 \mathrm{~km}$ from the city of Parakou. The Boko zone hospital is a preferred setting for the use of RDTs in accordance with national guidelines. This health center has a dispensary, a maternity ward, and a pediatric ward. In the laboratory, the sections mentioned above are also available and most of the examinations carried out at the Boko zone hospital are carried out at the HIA/CHU/Parakou.

\section{Study methods}

Type of study: This is a cross-sectional, descriptive and comparative study of the evaluative type, which aims to measure the performance of a test (RDT) in use for the diagnosis of malaria.

Sampling: The study focused on patients who came for consultation in the two health facilities. For patient identification, we used the non-probabilistic method and the convenience choice technique. Thus the sample size was 503 patients taken from among those received during our study period. To be included in our study, all patients of all ages and of both sexes must:

- Have been consulted at the General Medicine or in the other services of HIA/CHU Pkou and at the Boko zone hospital during the period of our study.

- Have been suspected of malaria by one of the prescribers of the aforementioned services and have received the following examinations: Hemoglobin level $(\mathrm{Hb})$ and / or thick gout plus parasite density.

- Prior to being submitted to a questionnaire in order to collect information relating to their identity, the clinical signs linked to malaria, the antimalarial drugs used, the last dose of the drug.

For the subjects in whom the clinical diagnosis of malaria is retained, we proceed to the venous or capillary sample for the subsequent manipulations.

Were excluded from our study, subjects admitted to health facilities for health reasons other than malaria, and patients who refused to participate in our study.

Data collection techniques and tools: Interview and observation are the two techniques we used to carry out this study.

The personal interview was used using a questionnaire to collect information from each patient.

The observation was made from an observation grid to assess the results of the microscopy and the RDT of each patient.

\section{Organization of data collection:}

It followed the following steps:

- An exchange session with health workers involved in collecting patient data took place on April 20, 2015 in Parakou. Data collection took place from April 21 to July 30, 2015

- All microscopies were done by technicians confirmed for the diagnosis of malaria and RDTs were performed in the same patients; 
- $\quad$ Patient information was collected by health workers.

Technical approach:

Blood smear/thick drop staining of slides and reading: We did a capillary swab in patients with only thick gout as requested and a venipuncture swab at the elbow crease for patients with EW/DP and other examinations. After installing the patient, and preparing the materials below, we welcome the patient for the type of examination.

Thick drops and blood smears were performed on the same slide for the identification and specification of the parasites using the Giemsa technique. Slides were stained and visualized using a 100 $\times$ oil immersion objective of a compound microscope. At least 100 fields were examined before a thick blot was considered negative. For positive slides, parasites were assessed and parasitaemia (density of (density of parasites) was determined by counting only asexuals out of 200 White Blood Cells (WBCs), then multiplying the number of parasite found by 8000 divided by 200 , assuming that the mean total number of mean total WBC of individuals is 8000 cells $/ \mu \mathrm{L}$ of blood [26].

The slides were read blind by two independent microscopists. Positive/negative cases were only called after confirmation by the two microscopists. Based on microscopy, parasite density estimates were calculated as the average of the values that were within the range of difference between the two readers. Two readings were considered to be divergent if their difference was outside the $95 \%$ of the agreement limits of the previous paired readings. The level of parasitaemia was recorded by parasite category/ $\mu \mathrm{L}$ of blood) [26].

\section{Strongly positive case:}

- $\quad$ Divide the field into four and count the pests in a quarter of the field.

- $\quad$ Count white blood cells throughout the field.

- Repeat the same in 10 fields; then calculate the parasite density as follows: (Training manual for medical biotechnologists, March 2012).

Carrying out the ToRs and results: Rapid Diagnostic Tests (RDTs) and microscopy on blood samples were tested for malaria parasites using SD Bioline Malaria Antigen Pf (HRP2).

\section{a) Technique for performing an RDT:}

The steps for carrying out a RDT:

\section{Preparatory phase:}

- $\quad$ Prepare all the material

- $\quad$ Observe the protective hygiene measures of the place;

- Let the kit components return to room temperature if necessary (if kept cool).

\section{Unfolding phase:}

- Check the color of the dissectant (according to the manufacturer's instructions).

- Identify the cassette/strip by writing the client's last name, first name and the date of the test in pencil.
- $\quad$ Collect with a micropipette a small drop of blood (about 5ul) already collected on the EDTA.

- Transfer the drop of blood collected to the well indicated in the package leaflet.

- $\quad$ Place two drops of the lysis solution (Buffer) in the second well indicated on the instructions.

- Take the reading after 15 to $20 \mathrm{~min}$, depending on the length of time specified by the manufacturer

- Dispose of handling waste in accordance with bio-medical waste management procedures.

- $\quad$ Follow the instructions specific to the type of RDT.

\section{b) Interpretation of SD Bioline malaria Ag. Pf test results}

For two-band RDTs such as SD Bioline malaria Ag. Pf used in our study, we distinguish:

- $\quad$ The control strip (C)

- $\quad$ Plasmodium falciparum identification test strip (T).

- A positive test results in the appearance of two distinct bands (one control band and the other P. f): positive test for plasmodium falciparum.

- A negative test results in the appearance of the only band in the control zone $(\mathrm{C})$

- The absence of a control strip invalidates the test, even if the test strip is present.

Note that the results of the microscopy and the RDT are entered into the investigation directly on the investigation form.

- Collect blood in an ETDA tube at the fold of the elbow, preferably using a suitable needle by first placing a tourniquet above the elbow.

\section{Criteria for choosing a Rapid Diagnostic Test}

The choice of an RDT must take into account its sensitivity, its specificity, its stability, its ease of use, its principle of antigen detection and its cost. The relevance of RDTs specific for P. falciparum or specific for other plasmodium species, and pan-specific tests varies according to the area of intervention and with the relative prevalence of the different human plasmodium species in the region [24].

Sensitivity (Se) of a diagnostic test: The sensitivity of a test is its ability to detect cases of a disease. To measure the sensitivity, it is necessary to have a group of patients selected by indisputable methods making it possible to certify the presence of the disease, which here is microscopy [24]. This is the proportion of true positives out of the total number of sick subjects.

\section{TP: True Positives, FN: False Negatives}

Specificity (Sp) of a diagnostic test: The specificity of a test is its ability to correctly identify individuals who are not affected by the disease [24].

In mathematical terms, specificity is the proportion of true negatives out of the total number of healthy subjects. 
Table 1: Summary table of performance indicators

\begin{tabular}{|c|c|c|c|c|}
\hline & & \multicolumn{2}{|c|}{ Microscopy } & \multirow{2}{*}{ Total } \\
\hline & & Positive & Negative & \\
\hline \multirow{2}{*}{ Test } & Positive & True Positive (TP) & False Positive (FP) & PV + FP Total Positive Tests \\
\hline & Negative & False Negative (FN) & True Negative (TN) & FN + TN Total Negative Tests \\
\hline Total & & TP + FN Total Patients & FP + TN Total Non-Sick & $P V+F P+F N+T N$ Total Workforce \\
\hline
\end{tabular}

Sensitivity: Se = PVI(PV + FN); Positive Predictive Value: VPP $=\mathrm{PV} /(\mathrm{PV}+\mathrm{FP})$. Specificity: $\mathrm{Sp}=\mathrm{TN} /(\mathrm{FP}+\mathrm{TN}) ;$ Negative Predictive Value: $N P V=\mathrm{TN} /(\mathrm{FN}+\mathrm{TN})$

Positive predictive value (PPV): The positive predictive value of a test is the probability of being ill when the test result is positive [24]

Negative predictive value (NPV): The negative predictive value of a test is the probability of being healthy when the test result is negative [24].

The Table 1 below summarizes the different formulas for sensitivity, specificity, negative and positive predictive values. Calculated according to the methods published by (Delacour et al. 2005) [25].

\section{Other criteria}

The kappa coefficient (k): We first calculate the expected agreement $(\mathrm{Ca})$ and the coefficient of agreement $(\mathrm{Cc})$.

$C a=\frac{[(T P+F N)(T P+F P)]+[(F P+T N)(F N+T N)]}{T^{2}}$

$\mathrm{T}=$ number of samples examined

The coefficient of concordance $(\mathrm{Cc})$ is equal to the sum of the matching results over the total number of exams. It is expressed as a percentage [24].

$\mathrm{Cc}=$ Number of matching exams/Number of compared exams

$K=\frac{C c-C a}{1-C a}$

It is expressed by a number between -1 and +1

- A K close to -1 means a complete mismatch.

- $\quad$ A K close to 0 means an average match due to chance.

- $\quad$ A K close to +1 means absolute agreement.

In biology, a correct $\mathrm{K}$ coefficient between two techniques must be greater than $0.8(80 \%)$ [24].

- $\quad$ False positive rate in individuals with the sign $=(\mathrm{FP} / \mathrm{TP}+$ $\mathrm{FP})$ in $\%$.

- False negative rate in individuals who do not have the sign $=(\mathrm{FN} / \mathrm{FN}+\mathrm{TN})$ in $\%$.

- Prevalence: rate of the disease in the entire study population $=(\mathrm{PT}+\mathrm{FN}) /(\mathrm{TP}+\mathrm{FP}+\mathrm{FN}+\mathrm{TN})$ in $\%$.

- $\quad$ Youden index $=$ sensitivity + specificity -1

- A negative value means that the test is ineffective.

- When the value of the index approaches 1 , it is concluded that the test is effective.

- Yule coefficient (Q): $\mathrm{Q}=\left(\mathrm{TP}^{\star} \mathrm{TN}-\mathrm{FP}^{\star} \mathrm{FN}\right) /$ $\left(\mathrm{TP}^{\star} \mathrm{TN}+\mathrm{FP}^{\star} \mathrm{FN}\right)$.

It measures the strength of the link between the two variables (reference test/test to be evaluated).

The strength of the bond is negligible if $\mathrm{Q}=(0.01-0.09)$; Slight if $\mathrm{Q}=(0.10-0.29)$; Moderate if $\mathrm{Q}=(0.50-0.69)$; Strong if $\mathrm{Q}=(0.50-0.69)$ and Very strong if $Q=(0.70-1)$.

\section{Ethical aspects}

Authorization to conduct the study was obtained from the Chief Medical Officer of the Parakou communal health center and the managers of the two laboratories (Boko zone hospital and HIA/ $\mathrm{CHU}$ ). The informed consent of each patient was obtained before their participation. The results of the study were free from any manipulation that could affect their quality.

\section{Data processing}

The data collected was entered and analyzed using software PPE INFO and Excel.

\section{Results}

General characteristics of patients: Table 2 below showing the distribution of patients according to age group, sex and religion indicates that patients under the age of 5 are the least represented (23.5\%) compared to elderly patients over five years. Of the 503 patients in our study, 264 (52.5\%) were male and 239 (47.5\%) female. Christians predominate other religions with a percentage of $48.7 \%$.

Comparison of the results of RDT with those of thick gout in the laboratory diagnosis of malaria: In Table 3, showing the distribution of patients according to the results of thick gout, we Table 2: Distribution of patients according to age group, sex and religion.

\begin{tabular}{|l|c|c|}
\hline \multicolumn{1}{|c|}{ Patient Characteristics } & Workforce (N=503) & Percentage (\%) \\
\hline Age group (in years) & & \\
\hline 0-1years & 29 & 5.8 \\
\hline 1 year-5 years & 89 & 17.7 \\
\hline 5-15 years & 62 & 12.3 \\
\hline$>15$ years & 323 & 64.2 \\
\hline Total & $\mathbf{5 0 3}$ & $\mathbf{1 0 0}$ \\
\hline Sex & & \\
\hline Male & 264 & 52.5 \\
\hline Female & 239 & 47.5 \\
\hline Total & $\mathbf{5 0 3}$ & $\mathbf{1 0 0}$ \\
\hline Religion & & \\
\hline Muslim & 174 & 34.6 \\
\hline Christian & 245 & 48.7 \\
\hline Traditional & 84 & 16.7 \\
\hline Total & $\mathbf{5 0 3}$ & $\mathbf{1 0 0}$ \\
\hline
\end{tabular}


Table 3: Distribution of patients according to the results of Microscopy.

\begin{tabular}{|c|c|c|}
\hline Thick gout Results & Workforce & Percentage (\%) \\
\hline Positive & 180 & 35.8 \\
\hline Negative & 323 & 64.2 \\
\hline Total & $\mathbf{5 0 3}$ & $\mathbf{1 0 0}$ \\
\hline
\end{tabular}

Table 4: Distribution of patients according to RDT results.

\begin{tabular}{|c|c|c|}
\hline RDT Results & Workforce & Percentage (\%) \\
\hline Positive & 199 & 39.6 \\
\hline Negative & 304 & 60.4 \\
\hline Total & $\mathbf{5 0 3}$ & $\mathbf{1 0 0}$ \\
\hline
\end{tabular}

Table 5: Sensitivity and specificity of RDT.

\begin{tabular}{|c|c|c|c|}
\hline \multirow{2}{*}{} & \multicolumn{2}{|c|}{ RDT } & \multirow{2}{*}{ Total } \\
\cline { 2 - 3 } & Positive & Negative & \\
\hline Positive & $165(91.70 \%)$ & 15 & 180 \\
\hline Negative & 34 & $289(89.50 \%)$ & 323 \\
\hline Total & $\mathbf{1 9 9}$ & $\mathbf{3 0 4}$ & $\mathbf{5 0 3}$ \\
\hline
\end{tabular}

Table 6: Sensitivity of RDT as a function of the parasite.

\begin{tabular}{|c|c|c|c|}
\hline $\begin{array}{c}\text { Parasitemia (Parasites } \\
\mu \mathrm{l})\end{array}$ & $\begin{array}{c}\text { Positive } \\
\text { Microscopy }\end{array}$ & RDT Positive & Sensitivity (\%) \\
\hline$[1-100]$ & 6 & 0 & 0 \\
\hline$[101-200]$ & 8 & 0 & 0 \\
\hline$[201-500]$ & 39 & 38 & 97.4 \\
\hline$[501-1000]$ & 50 & 50 & 100 \\
\hline$[1001-5000]$ & 35 & 35 & 100 \\
\hline$>5000$ & 42 & 42 & 100 \\
\hline Total & $\mathbf{1 8 0}$ & 165 & 91.7 \\
\hline
\end{tabular}

Table 7: Distribution of patients according to the existence or not of previous anti-malaria treatment.

\begin{tabular}{|c|c|c|}
\hline Previous Anti-malaria Treatment & Patients & Percentage\% \\
\hline Yes & 85 & $16.90 \%$ \\
\hline No & 418 & $83.10 \%$ \\
\hline Total & $\mathbf{5 0 3}$ & $100 \%$ \\
\hline
\end{tabular}

observed that 180 had their TG positive out of 503 patients who had a clinical diagnosis of malaria, i.e. a prevalence of $35.8 \%$ and 323 are negative, i.e. $64.2 \%$.

The RDTs carried out on the 503 patients showed that 199 cases were positive on the RDT, ie $39.6 \%$ against 304 , or $60.4 \%$ negative (Table 4).

In Table 5 showing the sensitivity and specificity of the RDT, we notice that out of a total of 180 confirmed samples (TG), 165 are positive for the RDT, which corresponds to a Sensitivity (Se) of $91.7 \%$. Out of a total of 323 thick film negative (TG) samples, 304 had their RDT negative; which corresponds to a Specificity (Sp) of $89.5 \%$ during our study.

By distributing the 180 samples positive to the reference test by class of parasitemia in Table 6, we find that the capacity for detecting malaria by the RDT (Sensitivity) is zero for the parasitemias between 1 and 200; it is $97,4 \%$ for parasitemias ranging from 201 to 500 and
Table 8: Distribution of patients on anti-malaria treatment according to the different molecules.

\begin{tabular}{|c|c|c|}
\hline Previous Anti-Malaria Treatment & Numbers (N = 85) & Percentage\% \\
\hline ACT & 46 & 54.1 \\
\hline QUININ 300mg & 39 & 45.9 \\
\hline TOTAL & $\mathbf{8 5}$ & 100 \\
\hline
\end{tabular}

Table 9: Paintings: Distribution of patients who received anti-malaria treatment according to Microscopy and RDT results.

\begin{tabular}{|c|c|c|c|}
\hline \multirow{2}{*}{ Microscopy } & \multicolumn{2}{|c|}{ RDT } & \multirow{2}{*}{ Total } \\
\cline { 2 - 4 } & Positive & Negative & \\
\hline Positive & $36(92.30 \%)$ & 3 & 39 \\
\hline Negative & 8 & $38(82.60 \%)$ & 46 \\
\hline Total & 44 & $\mathbf{4 1}$ & $\mathbf{8 5}$ \\
\hline
\end{tabular}

Table 10: Distribution of patients who did not receive antimalarial treatment according to Microscopy and RDT results.

\begin{tabular}{|c|c|c|c|}
\hline \multirow{2}{*}{ Microscopy } & \multicolumn{2}{|c|}{ RDT } & \multirow{2}{*}{ Total } \\
\cline { 2 - 4 } & Positive & Negative & \\
\hline Positive & $129(91.50 \%)$ & 12 & 141 \\
\hline Negative & 26 & $251(90.60 \%)$ & 277 \\
\hline Total & 155 & $\mathbf{2 6 3}$ & 418 \\
\hline
\end{tabular}

$100 \%$ for parasitemias greater than 501 .

In Table 7 below showing the distribution of patients according to the existence or not of previous antimalarial treatment, we found that out of the 503 patients in our study, 85 were on previous antimalarial treatment which represents a percentage of $16.9 \%$ against $83.1 \%$ who are without anti-malaria treatment.

In Table 8 showing the distribution of patients on anti-malaria treatment according to the different molecules, it was found that the patients used more quinines and ACT in the following respective proportions: $54.1 \%$ and $45.9 \%$.

In the distribution of patients who received anti-malaria treatment according to the results of TG and RDT in Table 9 below, we see that out of a total of 39 patients confirmed by Microscopy and who are under treatment, 36 are positive for RDT; which corresponds to a sensitivity of $92.3 \%$. In the same series of patients under treatment, we find that out of a total of 46 samples negative for thick gout, 38 are also negative on RDT, which corresponds to a specificity of $82.6 \%$.

Table 10 below shows the distribution of patients who did not receive antimalarial treatment according to the results of TG and the RDT. Out of a total of 141 patients confirmed by positive thick gout and without treatment, 129 were also confirmed by RDT, which corresponds to a sensitivity of $91.5 \%$ of positive by RDT. In the same series of patients without treatment, we find that out of a total of 277 thick-drip negative patients, RDT gave a negative result in 251 patients; which corresponds to a specificity of $90.6 \%$ for RDT in patients without treatment.

Table 11 below shows the summary of the results obtained in patients under treatment and without processing according to TG and RDT tests.

In Table 12 showing the Synthesis of the sensitivity and specificity 
Table 11: Summary of the results obtained in patients.

\begin{tabular}{|c|c|c|c|c|c|}
\hline \multirow{2}{*}{ Previous treatment } & Microscopy Negative & Microscopy Positive & Microscopy Negative & Microscopy Positive & \multirow{2}{*}{ Total } \\
\hline & Negative RDT & RDT Positive & RDT Positive & Negative RDT & \\
\hline Yes & 38 & 36 & 8 & 3 & 85 \\
\hline No & 251 & 129 & 26 & 12 & 418 \\
\hline Total & 289 & 165 & 34 & 15 & 503 \\
\hline
\end{tabular}

Table 12: Summary of the sensitivity and specificity of the RDT according to the category of patients.

\begin{tabular}{|c|c|c|}
\hline RDT Results & Sensitivity (\%) & Specificity (\%) \\
\hline Patients on Treatment & 92.3 & 82.6 \\
\hline Patients Without Treatment & 91.5 & 90.6 \\
\hline
\end{tabular}

Table 13: Summary of RDT performance indicators.

\begin{tabular}{|l|c|}
\hline \multicolumn{1}{|c|}{ RDT Performance Indicator } & Indication Value (\%) \\
\hline Sensitivity (Se) & 91.7 \\
\hline Specificity (SP) & 89.5 \\
\hline Positive Predictive Value (PPV) & 82.9 \\
\hline Negative Predictive Value (NPV) & 95 \\
\hline False Negative Rate & 4.9 \\
\hline False Positive Rate & 17.1 \\
\hline Concordance Coefficient (Cc) & 90 \\
\hline Kappa Coefficient (K) & 0.88 \\
\hline Youden Index & 0.81 \\
\hline Yule Coefficient & 0.97 \\
\hline
\end{tabular}

of the RDT according to the category of patients, we find that, the sensitivity of the RDT is practically the same for the two subgroups; but the specificity is $90.6 \%$ in patients without treatment against $82.6 \%$ in patients on treatment.

Table 13 below shows the summary of RDT performance indicators.

\section{Discussions}

From our work, the results obtained made it possible to assess all the variables provided for in the framework of this study. In view of the above, the objectives pursued were achieved and these results validate the RDTs SD Bioline Malaria Ag Pf in use as a relevant diagnostic tool in the management of malaria.

\section{Validity and quality of the results of the study}

During this study, carried out at the HIA-CHU/PKOU) and at the Boko zone hospital, we diagnosed 503 patients divided into six age groups. The sampling method and technique were applied and described in the protocol. The calculation of the performance indicators of the RDT in use, during this evaluation, was made in accordance with international standards [24]. Among the two health structures that served as a framework for the study, the Boko zone hospital is a center where most of the patients from Parakou refer. This undoubtedly participated in the recruitment of a significant proportion of patients with elevated parasitaemias.

For the present evaluative study of SD Bioline Malaria Ag Pf detecting the HRP2 protein, the method used as a reference is the thick film. In the different age and sex groups, the prevalence of malaria in Parakou is $39.6 \%$ in the SD Bioline Malaria Ag Pf test and $35.8 \%$ in thick gout. Our RDT test prevalence is lower than that reported by Denise Davou in 2011 [8] in Parakou, which was 47\%, on the other hand, it is higher than that reported by Hounto et al, in 2013 [9] in Cotonou, which was $25.4 \%$. Compared to thick gout, it is higher than that reported by some authors (Tokponnon et al in 2014 [27] in the plateau, Hounto et al, in 2013 [9] in Cotonou, which was respectively $(17 \%, 22.8 \%)$. It is approximately equal to the $34 \%$ found by the Demographic Health Survey (DHS) in 2012 [28] on children aged 6 to 56 months in the department of Borgou. However, all these results show the mesoendemic character of malaria in the locality.

\section{Comparison of the results of RDT with those of thick gout in the laboratory diagnosis of malaria}

The sensitivity and specificity of RDT: The sensitivity and specificity of RDT during our study were $91.7 \%$ and $89.5 \%$, respectively. In view of the work carried out on the performance of RDT, we noted that the sensitivity found in our study is lower than that found by other authors. Thus, Davou Denise in 2011 [8] in Parakou reported a sensitivity of $95.5 \%$, that of Hounto et al in 2013 [9] in Cotonou, is $96.3 \%$, as well as that reported by Munier et al in 2009 [30] in Senegal which was by $97 \%$. It is also lower than the 94.7\% reported by Dongmo Tanke Norbert in 2012 [10] in Cameroon with Diaspot -Malaria-Pf, a test also detecting the HRP2 protein and finally Abba, et al. in 2012 [31] that reported 93.2\% sensitivity in meta-analyzes. On the other hand, it is appreciably equal to the $92 \%$ reported by Chinkhumba et al. in 2010 [32].

As for the specificity, it is $89.5 \%$. This value obtained in our study seems low, reflecting a high number of false positive results but remains comparable to that of Munier et al. in 2009 [30], of Davou Denise in 2011 [8] in Parakou. On the other hand, it is lower than that reported by Abba, et al in 2012 [31], Hounto et al. in 2013 [9] in Cotonou who had respectively found (95.6\% and 96.7\%).

False positives: Our results revealed 34 cases of false positives, or $17.1 \%$. This rate is slightly higher than that found by Davou Denise which was $13.5 \%$ and that presented by the WHO in 2009 which was $4 \%$. During our study, we found that its false positives were from patients who had received previous antimalarial drugs before they were admitted to hospital. This observation was made in patients of the Boko zone hospital who presented high parasite densities. We also observed in patients collected after previous treatments with antimalarial drugs that the thick drops were negative while the RDTs were positive. These false positive cases could be due to the persistence of the HRP2 protein in the blood as recognized by several authors [3335]. Of the 34 false positive patients, only 8 were reported as being on treatment, and the 26 did not. Faced with this result, it appears that the false positive results are not only linked to the persistence of the HRP2 protein in the blood [11]. To do this, we asked ourselves 
whether these 26 remaining patients would not be in an economy of truth as to the status of prior antimalarial treatment or not; or would they not have self-medicated antimalarial (herbal tea and others)? We should also point out that during the collection of information for filling out our survey form, we experienced great difficulties in terms of understanding the language of some patients, which could induce poor reporting of patient opinions on previous treatment received as reported as the cause of false positives by Dongmo Tanke in his work carried out in 2012 [11]. In addition, other factors were cited as causes of false positives. Cross reactions with rheumatoid factors in patients without malaria [36], infections by Schistosoma mekongi proven by Leshem et al. 2011 [37], which can also explain these cases of false positives.

False negatives: Of a total of 180 TG confirmed specimens, 165 are RDT positive. The study found 15 cases of false negatives, or $4.9 \%$. This value is higher than that found by Davou Denise, which was $3.6 \%$ in his work carried out in Parakou in 2011. The distribution of the sensitivity of the RDT according to the parasitaemia allowed us to note that the 14 cases of false negatives correspond to cases of low parasitaemias. It thus appears a weak capacity of the RDT to detect the disease in the event of weak parasitaemia as notified by the WHO in its detection threshold indicated in the report of the results of the evaluations of the products, i.e. 200 parasites/ $\mathrm{LL}$ of blood (WHO 2012) and also reported by Munier et al. in 2009 [30]. On the other hand, we received 1 case with a parasite density greater than 400 parasites per $\mu \mathrm{L}$ of blood but whose RDT was negative. If the literature agrees that in general based RDTs (HRP2) have better sensitivities and therefore give relatively fewer false negatives, then an explanation would be needed in this particular case. Our case of false negatives with a parasite density greater than 400 , could be due to certain parasites which would not have produced Pf HRP2 and therefore would be responsible for most infections of the bloodstream and false negative results, such as reported to Mali by they give relatively fewer false negatives, so explanations would be needed in this particular case. Our case of false negatives with a parasite density greater than 400 , could be due to certain parasites which would not have produced Pf HRP2 and therefore would be responsible for most infections of the bloodstream and false negative results, such as reported to Mali by they give relatively fewer false negatives, so explanations would be needed in this particular case. Our case of false negatives with a parasite density greater than 400 could be due to certain parasites, which would not have produced Pf HRP2 and therefore would be responsible for most infections of the bloodstream and false negative results, such as reported to Mali by Koita et al. [38]. In addition, some authors have suggested the existence of an antigenic variation of the PfHRP2 gene, while the Plasmodium pLDH and aldolase genes do not [39-42].

Based on the RDT performance evaluation criteria, the Kappa coefficient gives 0.88 ; which means a good agreement of the RDT with the thick gout. This good performance is confirmed by the Youden index, which is 0.81 . Yude's Q coefficient, found at 0.97 , testifies to the very high intensity of the link between Thick Gout (TG) and RDT BIOLINE Malaria Ag Pf.

\section{Conclusion}

At the end of our work, we retain that the effectiveness of the treatment prescribed by the doctor depends largely on the result provided by the laboratory. The effectiveness of RDTs has limits in its incapacity to determine the parasitaemia of a patient and especially its insufficiencies in the detection of the disease in the event of weak parasitaemia are not negligible criteria. The purpose of the present study was to compare the results of RDT in use with that of thick gout in the diagnosis of malaria. Our study showed that the RDT SD BIOLINE used has a good performance in the diagnosis of Plasmodium infection. In a general way, the level of performance was judged to be good because it makes it possible to diagnose 9 cases out of 10 among patients suspected of malaria by presenting the following evaluation criteria: Sensitivity $91.7 \%$; specificity $89.5 \%$; positive predictive value $82.9 \%$; negative predictive value $95 \%$, kappa coefficient of 0.88 .

Therefore, we encourage the use of these RDTs in all health centers, both peripheral and referral health facilities to facilitate early decision-making. However, the use of these tests must necessarily be coupled with microscopy in these reference centers in order to ensure proper care.

\section{References}

1. World Health Organization, (Geneva). World Malaria Report. 2012.

2. World Health Organization, (Geneva). World Malaria Report. 2013.

3. Ministry of Health, Benin national strategic plan to fight against malaria. (2011-2015).

4. Bouzouaia Noureddine. Malaria in Tunisia. Technical guide for control and management. 2010.

5. World Health Organization, (Geneva). World Malaria Report. 2010.

6. World Health Organization, (Geneva). Malaria rapid diagnostic test performance review: WHO product trial series 1-3 (2008-2011).

7. Ministry of Health, Benin 2011 national malaria case management guidelines.

8. Davou Denise. Comparative study of the results of thick gout and the rapid diagnostic test (SD BIOLINE MALARIA Ag Pf) in the diagnosis of malaria at CHDU-B and CS of KPEBIE in Parakou. 2011.

9. Hounto D. Kinde-Gazard, C. Keke et al. Evaluation of a rapid diagnostic test and a portable fluorescence microscope for the diagnosis of malaria in Cotonou (Benin). 2013; 106: 27-31.

10. Dongmo Tanke Norbert. Comparative study of a Malaria Rapid Diagnostic Test (RDT) with Thick Gout (GE) at the Bafoussam regional hospital in Cameroon. 2012.

11. Mouatcho C, Goldring JP Dean. Malaria rapid diagnostic tests: challenges and perspectives. J Med Microbiol. 2013; 62.

12. Inserm - News $N^{\circ}$ 205. 2007.

13. Ministry of Public Health and Population, Central African Republic, MADJI Nestor "the major dates in the history of malaria".

14. Ministry of Health, Benin Health Statistics Yearbook. 2012.

15. French Association of Parasitology and Mycology Teachers (ANOFEL). 2014: 5-20.

16. Chabasse D, Danis M, Guiguen C. Malaria. In: parasitology and mycology of temperate and tropical regions. Paris: Ed Masson. 2007: 23-39.

17. Clara Loïzzo, Masséna, Nice, et al. Geography of health: spaces and societies. 2012.

18. Sustainable development and health, the fight against a major scourge malaria.

19. World Health Organization, (Geneva). Basic technique for the biological 
diagnosis of malaria. Geneva: WHO. 1994.

20. Hance Garnotel E, Depina J. Rapid immunochromatic tests for the detection of malaria, principles and strategies for use. Med Trop. 2005; 65: 394.

21. Bharti AR, Letendre SL, Patra KP, et al. Malaria diagnosis by a polymerase Chain Reaction based assay using a pooling strategy. Am J Trop Med Hyg 2009; 81: 754-757.

22. Management and prevention of imported Plasmodium falciparum malaria recommendations for clinical practice. 2007: 10.

23. Djeutchouang Sayang Collins. Interest of the use of rapid diagnostic tests for malaria on the cost and effectiveness of the management of febrile patients in Yaoundé, Cameroon.

24. Ancelle T. Performance of a technique. N: Epidemiological Statistics. $2^{\text {nd }}$ ed Paris: Maloine. 2006: 241-13.

25. Delacour H, Servonnet A, Perrot A, et al. "ROC (receiver Operating characteristics) curve: principles and application in biology." Ann Biol Clin (Paris). 2005; 63: 145-154.

26. Ministry of Health, (Benin). National malaria control policy and strategic implementation framework. Cotonou: NP. 2005: 50.

27. Tokponnon Filémon, Hounto Aurore, Yolande Sissinto, et al. Impact of long-lasting, insecticidal nets on anaemia and prevalence of Plasmodium falciparum among children under five years in areas with highly resistant malaria vectors. Malaria J 2014, 13:76.

28. Ministry of Health (Benin). Demographic and Health Survey. 2012.

29. Siala E, Ben R, Bouratbine A. News of the biological diagnosis of malaria. Tunisian Journal of Infectiology. 2010; 4: 5-9.

30. Munier A, Diallo A, Sokhna C, Chippaux JP. Evaluation of a rapid diagnostic test for malaria in rural health posts in Senegal. Med Trop. 2009; 69: 496-495.

31. Abba K, Deeks JJ, Olliaro PL, et al. Rapid diagnostic tests for the diagnosis of falciparum malaria in endemic countries Cochrane Data base Syst Rev. 2011; 7: CD008122.

32. Chinkhumba J, Skarbinski J, Chilima B, et al. Comparative field performance and adherence to test results of four rapid diagnostic tests for malaria in febrile patients over five years of age in Blantyre, Malawi. Malaria Journal. 2010; 9: 209.

33. Wongsrichanalai $\mathrm{C}$, Barcus $\mathrm{MJ}$, Muth $\mathrm{S}$, et al. A review of diagnostic tools for malaria: microscopy and rapid diagnostic tests (RDTs). Am J Trop Med Hyg. 2007; 77: 119-127.

34. Kyabayinze DJ, Tibenderana JK, Nassali M, et al. Placental Plasmodium falciparum malaria infection: operational precision of HRP2 rapid diagnostic tests in an endemic malaria setting. Malar J. 2011; 10: 306.

35. Kattenberg JH, Ochodo EA, Boer KR, et al. A systematic review and metaanalysis: rapid diagnostic tests versus histology of the placenta, microscopy and PCR for malaria in pregnant women. Malar J. 2011; 10: 321.

36. Iqbal J, Sher A, Rab A. Plasmodium falciparum histidine-rich protein 2-based immunocapture diagnostic assay for malaria: cross-reactivity with rheumatoid factors. J Clin Microbiol. 2000; 38: 1184-1186.

37. Leshem E, Keller N, Guthman D, et al. False-positive Plasmodium falciparum histidine-rich protein 2 immunocapture assay results for acute schistosomiasis caused by Schistosoma mekongi. J Clin Microbiol. 2011; 49: 2331-2332.

38. Koita OA, Doumbo OK, Ouattara A, et al. Rapid diagnostic tests, false negatives for malaria and removal of the histidine-rich repeat region of the HRP2 gene .Am J Trop Med Hyg. 2012; 86: 194-198.

39. Baker K, Deeks JJ, Olliaro PL, et al. Global sequence variation in histidinerich proteins 2 and 3 of Plasmodium falciparum: implications for performing rapid diagnostic tests for malaria. Malar J. 2010; 9: 129.

40. Gamboa D, Ho MF, Bendezu J, et al. A large proportion of $P$. falciparum isolates in the Amazon region of Peru lack PfHRP2 and pfhrp3: Implications for rapid diagnostic tests for malaria PLoS ONE. 2010; 5: e8091.

41. Houzé S, Boly MD, Boly MD, et al. Combined deletions of PfHRP2 and pfhrp3 genes result in Plasmodium falciparum malaria false negative rapid diagnostic test. J Clin Microbiol. 2011; 49: 2694-2696.

42. Kumar N, Pande V, Bhatt RM, et al. Genetic deletion of HRP2 and HRP3 in Indian Plasmodium falciparum population and false negative malaria rapid diagnostic test. Acta Trop. 2013; 125: 119-121. 